\title{
Flow Tones in a Pipeline-Cavity System: Effect of Pipe Asymmetry
}

D. Erdem, D. Rockwell, P. Oshkai, M. Pollack

This report was prepared as an account of work sponsored by the United States Government. Neither the United States, nor the United States Department of Energy, nor any of their employees, nor any of their contractors, subcontractors, or their employees, makes any warranty, express or implied, or assumes any legal liability or responsibility for the accuracy, completeness or usefulness of any information, apparatus, product or process disclosed, or represents that its use would not infringe privately owned rights. 


\title{
FLOW TONES IN A PIPELINE-CAVITY SYSTEM: EFFECT OF PIPE ASYMMETRY ${ }^{*}$
}

\author{
by \\ D. Erdem* \\ D. Rockwell" \\ P. Oshkai* \\ M. Pollack ${ }^{\dagger}$ \\ *Lehigh University \\ ${ }^{\dagger}$ Lockheed-Martin \\ Bethlehem, PA
}

Flow tones in a pipeline-cavity system are characterized in terms of unsteady pressure within the cavity and along the pipe. The reference case corresponds to equal lengths of pipe connected to the inlet and outlet ends of the cavity. Varying degrees of asymmetry of this pipe arrangement are investigated. The asymmetry is achieved by an extension of variable length, which is added to the pipe at the cavity outlet. An extension length as small as a few percent of the acoustic wavelength of the resonant mode can yield a substantial reduction in the pressure amplitude of the flow tone. This amplitude decrease occurs in a similar fashion within both the cavity and the pipe resonator, which indicates that it is a global phenomenon. Furthermore, the decrease of pressure amplitude is closely correlated with a decrease of the Q (quality) -factor of the predominant spectral component of pressure. At a sufficiently large value of extension length, however, the overall form of the pressure spectrum recovers to the form that exists at zero length of the extension.

Further insight is provided by variation of the inflow velocity at selected values of extension length. Irrespective of its value, both the magnitude and frequency of the peak pressure exhibit a sequence of resonant-like states. Moreover, the maximum attainable magnitude of the peak pressure decreases with increasing extension length.

\section{INTRODUCTION}

Flow through a pipeline-cavity system represents a generic configuration occurring in a wide variety of applications. Due to the separation of flow along the cavity, the potential exists for coupling between the inherent instability mode of the cavity shear layer and an acoustic mode of the pipeline-cavity system. Even when the inflow to the cavity is fully turbulent, and the acoustic wavelength is much longer than the cavity length, pronounced flow tones can occur (Rockwell, Lin, Oshkai, Reiss and Pollack, 2001). In their investigation, the pipes on either side of the cavity were of the same length and

\footnotetext{
"Submitted to Journal of Fluids and Structures April 16, 2001; revised version submitted
} 
oscillations tended to occur only for the even acoustic modes of the pipeline-cavity system.

Flow tones in a pipeline-cavity system are expected to have certain features in common with tones generated by jet excitation of a long organ pipe (Cremer and Ising, 1967; Fletcher, 1979), jet flow through sequential orifice plates (Wilson, Beavers, DeCoster, Holger and Regenfuss, 1971; Flatau and Van Moorham, 1990; and Hourigan, Welsh, Thompson and Stokes, 1990), the wake from a flat plate in a test section (Parker, 1966; Cumpsty and Whitehead, 1971; Stoneman, Hourigan, Stokes and Welsh, 1988), a shear-layer past a cavity resonator (DeMetz and Farabee, 1977; Elder, 1978; Elder, Farabee and DeMetz, 1982; Nelson, Halliwell and Doak, 1981, 1983), and a cavity shear layer adjacent to a side branch duct/pipe (Pollack, 1980; Bruggeman, Hirschberg, van Dongen, Wijnands and Gorter, 1989, 1991; Ziada and Bühlmann, 1992; Kriesels, Peters, Hirschberg, Wijnands, Iafrati, Riccardi, Piva and Bruggemann, 1995; and Ziada and Shine, 1999). Selected features of these extensive investigations over the past three decades are briefly summarized by Rockwell, Lin, Oshkai, Reiss and Pollack (2001).

Directly pertinent to the present work are the tones arising in a pipeline-cavity system, which includes the case where the pipe actually protrudes into the cavity. These investigations were carried out at the University of Southampton and are described in a series of investigations, which extend from Davies (1981) to Davies (1996a,b). Pressure spectra showed a number of pronounced peaks, which indicated the generation of flow tones. For the case of a long pipe terminated by an axisymmetric cavity, Rockwell and Schachenmann $(1982,1983)$ employed linearized, inviscid stability theory to determine the phase shifts and amplitude spikes across the shear layer and, in addition, characterized the overall phase difference through the cavity during the generation of locked-on flow tones. Rockwell and Karadogan (1982) employed zero-crossing statistics, along with recursive digital filtering, to determine the degree of phase fluctuation of the organized oscillations of turbulent flow through the cavity.

For the configuration of the pipeline-cavity arrangement, which was investigated by Rockwell, Lin, Oshkai, Reiss and Pollack (2001), the effect of asymmetry, i.e., different lengths of the pipes attached to the inlet and outlet of the cavity, has not been addressed. The following issues therefore remain unclarified: 
(a) For the symmetrical pipeline-cavity system, flow tones occur only for even numbered acoustic modes of the pipeline-cavity resonator. This observation suggests that the occurrence of a pressure node, i.c., a velocity antinode, at the location of the cavity is an essential condition for the generation of a locked-on flow tone. A small asymmetry of the pipe arrangement, which corresponds to different lengths of the pipes at the inlet and outlet of the cavity, may effectively detune the locked-on flow tone by shifting the location of the pressure node. The possibility of a substantial reduction in pressure amplitude with a small extension of, for example, the outlet pipe has not been addressed.

(b) If a substantial reduction in the pressure amplitude can be attained, the issue arises as to whether it occurs for a pipe extension length that is a small fraction of the acoustic wavelength of the resonant mode.

(c) The consequence of variation of the inflow velocity at a given length of the pipe extension has remained unexplored. The possibility of generation of a sequence of resonant-like states of the magnitude and frequency of the peak pressure amplitude has not been pursued.

(d) The occurrence of locked-on flow tones is represented by a sharp peak in the pressure spectrum. The effect of the pipe extension on the shape of this peak, more explicitly its quality (Q)-factor, has not been addressed.

(e) For all of the foregoing variations of the peak pressure amplitude, the degree to which they occur globally, i.e., simultaneously at locations within the cavity and within the pipe resonator, has not been addressed.

\section{EXPERIMENTAL SYSTEM AND TECHNIQUES}

Complete details of the experimental system are described by Rockwell, Lin, Oshkai, Reiss and Pollack (2001). Only the essential features are described herein.

Air was supplied from an air compressor, through air treatment and accumulation facilities, located in a separate room, and isolated from the pipeline-cavity system. An inlet plenum served as a settling chamber at the upstream of the pipeline-cavity system. It was lined with acoustic damping foam and contained a honeycomb arrangement. The main pipeline-cavity arrangement consisted of two $7.87 \mathrm{ft}$. segments of 1 in. ID aluminum pipe. The length of the inlet pipe was selected to ensure that the boundary layer at the leading-edge of the cavity was fully-developed. One pipe segment was 
located on the upstream side of the Plexiglas cavity and the other on the downstream side. The pipe asymmetry was achieved by a pipe extension at the exit of the downstream pipe. This extension had an ID of 0.96 in. and a wall thickness of 0.02 in. The length of its protrusion from the main pipe was continuously varied from 0 in. to $24.49 \mathrm{in}$. by use of one of three extension pieces lengths: $4.0 \mathrm{in}, 14.0 \mathrm{in}$, and $26.0 \mathrm{in}$. The cavity subsystem for the present experiments, which is shown in Figure 1, had an internal diameter of 2 in., thereby providing a dimensionless cavity depth $\mathrm{W}^{*}=\mathrm{W} / \mathrm{D}=0.5$. The cavity length $\mathrm{L}$ was maintained constant at $\mathrm{L}=2.5$ in., corresponding to a dimensionless value $\mathrm{L}^{*}=$ $\mathrm{L} / \mathrm{D}=2.5$

As indicated in Figure 1, pressure transducer $\mathrm{p}_{\mathrm{a}}$ was located within the pipe upstream of the cavity, and transducers $\mathrm{p}_{b}$ and $\mathrm{p}_{c}$ were positioned within the cavity. High sensitivity PCB transducers (Model U103A02) were employed. The transducer outputs were connected to a PCB Piezotronic multi-channel signal conditioner, Model 48A. In order to prevent aliasing effects, a low-pass filter was applied to all pressure signals. The signals were then transmitted to a National Instruments board, Model PCI-MI0-16-E-4. This board sampled at a rate of 250,000 samples per second, which is well above the requirements of the present experiment. The acquisition of multiple data was accomplished using a multiplexing technique with a scan interval of approximately 32 microseconds. A Pentium II $350 \mathrm{MHz}$ computer and LabView software were used for processing the transducer signals. During acquisition of data, the sampling rate was set to 4,096 samples per second, which resulted in a Nyquist frequency of 2,048 Hz. Each of the spectra shown here was obtained by averaging a total of 42 data sets.

\section{EFFECT OF VARIATIONS OF LENGTH OF PIPE EXTENSION}

The three-dimensional plots of Figure 2 show the magnitude of the pressure peak on a plane of frequency $f$ versus dimensionless pipe extension length $X_{T} / \lambda$. The acoustic wavelength $\lambda$ is employed as a normalization length. This wavelength is $\lambda=\mathrm{c} / \mathrm{f}$, in which $c$ is the speed of sound and $f$ is the frequency of the locked-on flow tone for the case $X_{T}=0$. The speed of sound corresponding to $21^{\circ} \mathrm{C}$ and $70 \%$ relative humidity is $c=1,131.53 \mathrm{ft} / \mathrm{sec}$. Therefore, the value of $\lambda$ at the reference frequency of $\mathrm{f}=630 \mathrm{~Hz}$ was found to be $1.80 \mathrm{ft}$. Each of the three-dimensional plots corresponds to a different location of the pressure transducer. As defined in Figure 1, pressure $\mathrm{p}_{\mathrm{a}}$ is measured in the 
pipe resonator at a location upstream of the cavity, pressure $p_{b}$ on the floor of the cavity, and $\mathrm{p}_{\mathrm{c}}$ at the impingement corner of the cavity. The data presented in Figures 2 through 6 correspond to the constant inflow velocity of $130 \mathrm{ft} / \mathrm{sec}$.

Each of the plots of Figure 2 shows that the initial increase of $\mathrm{X}_{\mathrm{T}} / \lambda$ produces a decrease in the pressure amplitude $p$, then at a sufficiently large value of $X_{T} / \lambda$, the amplitude $\mathrm{p}$ again increases. At approximately $\mathrm{X}_{\mathrm{T}} / \lambda=0.5$, the amplitude $\mathrm{p}$ nearly recovers to its original value at $\mathrm{X}_{\mathrm{T}} / \lambda=0$. The scale of the pressure amplitude $\mathrm{p}_{\mathrm{a}}$ is a factor of 5 smaller than the scales on the plots of $\mathrm{p}_{\mathrm{b}}$ and $\mathrm{p}_{\mathrm{c}}$, in order to illustrate the similarity in the form of the pressure amplitude response at different locations. The general form of the response is the same at all locations, which indicates that the phenomenon is global. That is, the oscillations of the shear layer along the cavity and the acoustic resonance of the pipeline-cavity system are globally-coupled, and attenuation of the pressure amplitude $\mathrm{p}$ at one location is necessarily linked to attenuation at other locations as well.

Plan views of the two-dimensional plots are given in Figure 3. All of these views have a generally similar form. The nominal frequency of interest is the predominant resonant mode at approximately $630 \mathrm{~Hz}$. As the value of $\mathrm{X}_{\mathrm{T}} / \lambda$ increases from zero, the pressure amplitude decreases and, correspondingly, the frequency decreases slightly as well. At approximately $\mathrm{X}_{\mathrm{T}} / \lambda=0.25$, the magnitude of $\mathrm{p}$ reaches its lowest value, which is evident in both the lower and upper branches of the shaded pattern. Further increases of $\mathrm{X}_{\mathrm{T}} / \lambda$ yield an increase in pressure amplitude $\mathrm{p}$ along the upper branch until a peak value is again attained at approximately $\mathrm{X}_{\mathrm{T}} / \lambda=0.5$. In fact, this same pattern of pressure amplitude p occurs for the resonant modes located below and above the mode of interest, which is nominally at $630 \mathrm{~Hz}$. These modes have significantly lower values of pressure amplitude.

The plots of Figure 4 show the frequency $f_{P}$, the magnitude $P$ of the peak pressure (normalized by its maximum value $\mathrm{P}_{\max }$ ), and the $\mathrm{Q}$ (quality) -factor of the pressure peak as a function of length $X_{T}$ of the pipe extension, which is normalized by the pipe diameter $\mathrm{D}$ and the acoustic wavelength $\lambda$. These distributions correspond to pressure 
transducer $\mathrm{p}_{\mathrm{a}}$. They were constructed from the data exhibited in the top plots of Figures 2 and 3

The variation of $f_{P}$ in Figure 4 shows a maximum decrease of 4 percent at $X_{T} / \lambda=0.25$. This decrease is evident along the lower branch of pressure $p_{a}$ shown in Figure 3. It compares with a 6.5 percent decrease predicted from the simple theoretical relationship $\mathrm{f}_{\mathrm{n}}=\mathrm{nc} / 2 \mathrm{~L}_{\mathrm{T}}$, in which $\mathrm{L}_{\mathrm{T}}$ is the total length of the pipeline-cavity resonator with the extension $\mathrm{X}_{\mathrm{T}}$. For $\mathrm{X}_{\mathrm{T}} / \lambda>0.25$, the upper branch of $\mathrm{p}_{\mathrm{a}}$ in Figure 3 is considered. The value of $f_{P}$ in this region is higher than the nominal frequency $f_{P}=630 \mathrm{~Hz}$ of the predominant resonant mode. The value of $630 \mathrm{~Hz}$ is approached, however, at approximately $\mathrm{X}_{\mathrm{T}} / \lambda=0.5$.

The corresponding variation of the pressure magnitude $\mathrm{P} / \mathrm{P}_{\max }$ given in the middle plot of Figure 4, where $P_{\max }$ refers to the maximum value of pressure $P$ observed for the indicated range of $X_{T} / D$, shows a decrease of two orders of magnitude at $X_{T} / \lambda \cong 0.25$. $A$ substantial decrease of $\mathrm{P} / \mathrm{P}_{\max }$ is attainable, however, at a small value of $\mathrm{X}_{\mathrm{T}} / \lambda$. For example, $\mathrm{P} / \mathrm{P}_{\max }$ decreases to approximately one-third its initial value at approximately $\mathrm{X}_{\mathrm{T}} / \lambda=0.05$, which corresponds to $\mathrm{X}_{\mathrm{T}} / \mathrm{D}=1.25$ and $\mathrm{X}_{\mathrm{T}} / \mathrm{L}=0.5$, in which $\mathrm{L}$ is the cavity length and $\mathrm{D}$ is the pipe diameter. On the other hand, at $\mathrm{X}_{\mathrm{T}} / \lambda \cong 0.5$, the value of $\mathrm{P} / \mathrm{P}_{\max }$ recovers to a value close to the value at $X_{\mathrm{T}} / \lambda=0$. A value of $X_{\mathrm{T}} / \lambda=0.5$ corresponds to an extension of the standing wave pattern by one-half wavelength and recovery of the value of $\mathrm{P} / \mathrm{P}_{\max }$.

The corresponding variation of the quality factor $Q$, which is exhibited in the bottom plot of Figure 4, indicates that changes of $\mathrm{P} / \mathrm{P}_{\max }$ are directly linked to changes in $\mathrm{Q}$. The plot of $\mathrm{Q}$ indicates the occurrence of a locked-on flow tone at a zero value of extension, i.e. $X_{T} / D=X_{T} / \lambda=0$, in accord with the findings of Rockwell, Lin, Oshkai, Reiss and Pollack (2001). In that investigation, values of $Q=10^{3}$ corresponded to occurrence of a locked-on flow tone. This value is approximately one order of magnitude larger than the minimum value of $Q$ in the plot of Figure 4. In fact, the minimum values of $Q \cong 80$ to 100 are in the general range of the $Q$-factor of the pipeline-cavity system in absence of throughflow, as determined by transient decay from abrupt termination of 
loudspeaker excitation. These experiments were performed during a preliminary phase of this research program. This observation suggests that locked-on flow tones do not exist at, and in the vicinity of, $\mathrm{X}_{\mathrm{T}} / \lambda=0.25$.

Actual spectra are shown for selected values of $X_{T} / D$ in Figure 5 . It should be noted that plots of pressure amplitude vs. frequency are referred to as spectra throughout this article. The relation between the values of $\mathrm{X}_{\mathrm{T}} / \lambda$ and $\mathrm{X}_{\mathrm{T}} / \mathrm{D}$ can be obtained by comparison of the horizontal axes of the plots of Figure 4. At a sufficiently large value of $\mathrm{X}_{\mathrm{T}} / \mathrm{D}=1.5$, the peak amplitude of the predominant spectral component becomes substantially smaller and, at $\mathrm{X}_{\mathrm{T}} / \mathrm{D}=2.25$, decreases further. Moreover, the peak values of the side modes also decrease. This observation suggests that the effect of the pipe extension $X_{T}$ is to attenuate both the predominant locked-on mode and the neighboring modes that are excited by: turbulence within the pipes; and turbulence/instabilities along the shear layer of the cavity. The spectra given in the right column of Figure 5 show the recovery of the spectral distribution of pressure to approximately its original form for the case of no pipe extension. This recovery is evident from comparison of the spectral distributions at $X_{T} / D=0, X_{T} / D=9.25$, and 10. Portions of the spectra at $X_{T} / D=9.25$ and 10 , particularly in the vicinity of $450 \mathrm{~Hz}$ and $1,000 \mathrm{~Hz}$, do not, however, exhibit the same form as the spectrum at $\mathrm{X}_{\mathrm{T}} / \mathrm{D}=0$. Otherwise, the form of the recovered spectrum is quite similar, including occurrence of the first harmonic at approximately $1250 \mathrm{~Hz}$.

Figure 6 emphasizes the variation in shape of the predominant spectral component of each of the spectra of Figure 5. For purposes of comparison, the scale on the vertical ordinate of each plot of Figure 6 is adjusted to produce approximately the same peak amplitude. The increase in width of the spectral peak, which corresponds to a decrease in the value of the $Q$ (quality) -factor, is evident as $X_{T} / D$ increases from 0 to 2.25 . Conversely, decreases in the width of the peak over the range $X_{\Upsilon} / D=7.25$ to 9.25 correspond to increases of the value of $\mathrm{Q}$. At $\mathrm{X}_{\mathrm{T}} / \mathrm{D}=10.0$, the width of the spectral peak again decreases, due largely to the uncertainty of the Q-factor calculation, which is highly sensitive to the frequency resolution. Nevertheless, the value of $Q$ remains sufficiently high that the tone remains locked-on. That is, $Q$ is well above the aforementioned maximum values of $Q \cong 80$ to 100 . 


\section{EFFECT OF VARIATIONS OF INFLOW VELOCITY AT CONSTANT VALUE OF PIPE EXTENSION LENGTH}

The data described in the foregoing section show the substantial changes in frequency $f_{P}$, peak amplitude $\mathrm{P} / \mathrm{P}_{\max }$ and quality factor $\mathrm{Q}$ with changes in length of the pipe extension $X_{T}$ at a given value of inflow velocity $U$. In this section, emphasis is on the variation of the inflow velocity $U$ at selected values of the extension length $X_{\mathrm{T}}$.

The three-dimensional plots of Figure 7 correspond to values of $X_{\mathrm{T}} / \mathrm{D}=0.25$ and 1.5. Note that the vertical scale of the plot at $X_{T} / D=0.25$, which represents the amplitude of the pressure $\mathrm{p}$, has been compressed by a factor of four relative to the vertical scale at $X_{\mathrm{T}} / \mathrm{D}=1.5$. This compression allows direct comparison of the general form of the plots at $X_{T} / D=0.25$ and 1.5. It is evident that the overall response of the pressure amplitude $p$ and the frequency $f_{P}$ with variation of the inflow velocity $U$ is generally similar for both cases, despite the substantially lower magnitudes of $\mathrm{p}$ at $\mathrm{X}_{\mathrm{T}} / \mathrm{D}=1.5$. In essence, both plots show a similar, stage-like behavior. That is, the oscillation jumps to successively higher resonant modes of the pipeline-cavity system as velocity $U$ increases. Moreover, within each resonant mode, a relatively large value of pressure $\mathrm{p}$ is attained; near the jump from one mode to the next, the value of pressure $\mathrm{p}$ becomes relatively small.

These features are shown more explicitly in the plots of Figure 8, which exhibit the peak pressure amplitude $P$ and the corresponding frequency $f_{p}$ taken from the spectrum at each value of $U$. At values of $X_{T} / D=0.25,1.0$ and 1.5 , the values of $P$ occur at approximately the center of each of the resonant modes of the plot of $f_{P}$, which is exhibited in the right column. In fact, this general tendency is discernible at the largest value of $X_{T} / D=2$, even though this is not a case of true lock-on, as is evident from the low values of $P / P_{\max }$ and $Q$ shown in the plots of Figure 4.

Figure 9 shows the data of Figure 8 superposed on common axes of $P$ versus $U$ and $f_{P}$ versus $U$. The legend that defines the symbols for values of $X_{T} / D$ is valid for both plots. In the top plot, two sets of symbols are filled, in order to allow better distinction between variations of $P$ versus $U$. The maximum values of $P$ shift to lower values of inflow velocity $U$ as $X_{T} / D$ increases from 0.25 to 1.0 . Further increases of $X_{T} / D$ result in 
either an increase, or an indiscernible change, of the velocity $U$ at which peaks of $P$ occur. Regarding the frequency $f_{P}$, the value of $X_{T} / D$ has a detectable effect on the value of $U$ at which the frequency jumps to the next mode. It varies over the range $131 \leq \mathrm{U} \leq$ $134 \mathrm{ft} / \mathrm{sec}$.

Selected spectra of pressure $p$, which correspond to the data sets of Figures 8 and 9, are given in Figure 10. For each spectrum, the values of $X_{\mathrm{T}} / \mathrm{D}$ and $U$ are indicated. The criterion for selection of the value of $U$ is as follows. It corresponds to the first peak amplitude $\mathrm{P}$ in Figure 8. The general form of the spectra of Figure 10 is quite similar. At the largest value of $\mathrm{X}_{\mathrm{T}} / \mathrm{D}=2.0$, attenuation of the peak at the predominant component of $680 \mathrm{~Hz}$ is accompanied by disappearance of the distinctive first harmonic at $1360 \mathrm{~Hz}$.

Zoomed-in plots of the predominant spectral peaks of Figure 10 are exhibited in Figure 11. Note that the vertical ordinate of each plot has been adjusted to provide the same peak amplitude, thereby providing a physical portrayal of the decrease in $Q$ factor with increasing $\mathrm{X}_{\mathrm{T}} / \mathrm{D}$. As in Figure 10, each of these zoomed-in plots is taken at the value of velocity $U$ corresponding to the maximum value of the peak pressure amplitude $P$. This observation indicates that, irrespective of the variation of velocity $U$ over a relatively wide range in Figures 7 through 9, the maximum value of the $Q$-factor is limited by the value of $X_{T} / D$. That is, at successively larger values of $X_{T} / D$, the maximum attainable value of $Q$ decreases.

\section{CONCLUDING REMARKS}

Flow through a pipeline-cavity system, where the length of the pipe at the cavity inlet is equal to the length at the outlet, is known to give rise to locked-on flow tones. The present investigation has examined the consequences of a difference in length of the inlet and outlet pipes; this was accomplished using an extension of length $X_{\mathrm{T}} / \mathrm{D}$ of the outlet pipe. The effects of this extension are summarized below.

(1) Substantial attenuation of the peak pressure amplitude of a locked-on flow tone occurs when the pipe extension $\mathrm{X}_{\mathrm{T}}$ is of the order of a few percent, e.g., five percent, of the acoustic wavelength, $\mathrm{X}_{\mathrm{T}} / \lambda=0.05$. This extension corresponds, however, to approximately one-half the cavity length $\mathrm{L}$, i.e., $\mathrm{X}_{\mathrm{T}} / \mathrm{L} \cong 0.5$.

(2) A reduction in the peak pressure amplitude $\mathrm{p}$ is associated with a corresponding decrease of the quality factor $\mathrm{Q}$ of the predominant pressure peak, as well as a 
decrease in the frequency $f_{\mathrm{P}}$ of the spectral component corresponding to the peak pressure. In fact, at sufficiently large values of $X_{T} / \lambda$, the values of $Q$ are approximately equal to the values obtained for turbulent flow through a pipe in absence of a cavity. This observation indicates that even a marginally locked-on state does not exist in this range.

(3) At a sufficiently large value of the pipe extension, corresponding to $X_{T} / \lambda \cong 0.5$, the amplitude of the peak pressure $p$, its frequency $f_{P}$, and the quality factor $Q$ approximately recover to the values obtained for no pipe extension, $\mathrm{X}_{\mathrm{T}} / \mathrm{D}=0$. Moreover, the overall form of the pressure spectrum is generally similar at $\mathrm{X}_{\mathrm{T}} / \lambda \cong 0.5$ and 0 .

(4) Variation of the inflow velocity $U$ at a given value of pipe extension $X_{\mathrm{T}} / \lambda$ yields a sequence of resonant-like states of the peak pressure amplitude. The major features of this sequence of states are successive jumps to higher order modes of the resonant pipeline-cavity system, as well as occurrence of a well-defined maximum of the peak pressure amplitude within each state. This stage-like behavior persists even for larger values of $\mathrm{X}_{\mathrm{T}} / \lambda$ for which the peak pressure amplitude is substantially attenuated. In essence, the effect of the pipe extension is to limit the value of peak pressure amplitude that can be attained when the inflow velocity $U$ is varied over a substantial range. The overall forms of the pressure amplitude and frequency variations with inflow velocity $U$ are generally similar at different values of $X_{T} / \lambda$.

\section{ACKNOWLEDGEMENTS}

The authors are grateful for financial support under Office of Naval Research Grant No. N00014-98-1-0817. 


\section{REFERENCES}

BRUGGEMAN, J. C., HIRSCHBERG, A., VAN DONGEN, M. E. H., WIJNANDS, A. P. J. \& GORTER, J. 1989 Flow induced pulsations in gas transport systems: analysis of the influence of closed side branches. Journal of Fluids Engineering 111, 484-491.

BRUGGEMAN, J. C., HIRSCHBERG, A., VAN DONGEN, M. E. H., WIJNANDS, A. P. J. \& GORTER, J. 1991 Self-sustained aero-acoustic pulsations in gas transport systems: experimental study of the influence of closed side branches. Joumal of Sound and Vibration 150, 371-393.

CREMER, L. \& ISING, H. 1967/68 Die selbsterregte schwingungen von orgelpfeifen. Acustica 19, 143-153.

CUMPSTY, N. S. \& WHITEHEAD, D. S. 1971 The excitation of acoustic resonances by vortex shedding. Journal of Sound and Vibration 18, 353-369.

DAVIES, P. O. A. L. 1981 Flow-acoustic coupling in ducts. Journal of Sound and Vibration 77, 191-209.

DAVIES, P. O. A. L. 1996a Piston engine intake and exhaust system design. Journal of Sound \& Vibration 190, 677-712.

DAVIES, P. O. A. L. 1996b Aeroacoustics and time varying systems, Journal of Sound and Vibration 190, 345-362.

DEMETZ, F. C. \& FARABEE, T. M. 1977 Laminar and turbulent shear flow-induced resonances. AIAA Paper 77-1293.

ELDER, S. A. 1978 Self-excited depth-mode resonance for a wall-mounted cavity in turbulent flow. Joturnal of Acoustical Society of America 64, 877-890.

ELDER, S. A., FARABEE \& T. M., DEMETZ, F. C. 1982 Mechanisms of flow-excited cavity tones at low Mach number. Journal of Acoustical Society of America 72, 532549.

HOURIGAN K., WELSH, M. C., THOMPSON, M. C. \& STOKES, A. N. 1990 Aerodynamic sources of acoustic resonance in a duct with baffles. Journal of Fluids and Structures 4, 345-370.

FLATAU, A. \& VAN MOORHAM, W. K. 1990 Prediction of vortex shedding responses in segmented solid rocket motors. AIAA Paper 90-2073.

FLETCHER, N. H. 1979 Airflow and sound generation in musical wind instruments. Annual Review of Fluid Mechanics 11, 123-146. 
KRIESELS, P. C., PETERS, M. C. A. M., HIRSCHBERG, A., WIJNANDS, A. P. J., IAFRATI, A., RICCARADI, G., PIVA, R. \& BRUGGEMANN, J.-C. 1995 High amplitude vortex-induced pulsations in a gas transport system. Journal of Sound and Vibration 184, 343-368.

NELSON, P. A., HALLIWELL, N. A. \& DOAK, P. E. 1981 Fluid dynamics of a flow excited resonance. Part I: Experiment. Journal of Sound and Vibration 78, 15-38.

NELSON, P. A., HALLIWELL, N. \& DOAK, P. E. 1983 Fluid dynamics of a flow excited resonance. Part II: Flow acoustic interaction. The dissipation of sound at an edge. Journal of Sound and Vibration 91, 375-402.

PARKER, R. 1966 Resonance effects in wake shedding from parallel plates: some experimental observations. Journal of Sound and Vibration 4, 62-72.

POLLACK, M. L. 1980 Flow-induced tones in side-branch pipe resonators. Journal of the Acoustical Society of America 67, 1153-1156.

ROCKWELL, D. \& KARADOGAN, H. 1982 Oscillations of an impinging turbulent jet: Coherence characterization via conditional sampling. Journal of Sound and Vibration 83 , No. 1, pp. 111-124.

ROCKWELL, D., LIN, J.-C., OSHKAI, P., REISS, M. \& POLLACK, M. 2001 Shallow cavity flow tone experiments: Onset of locked-on states. Submitted to Journal of Fluids and Structures.

ROCKWELL, D. \& SCHACHENMANN, A. 1982 Self-generation of organized waves in an impinging turbulent jet at low Mach numbers. Journal of Fluid Mechanics 117, 425-441.

ROCKWELL, D. \& SCHACHENMANN, A. 1983 The organized shear layer due to oscillations of a turbulent jet through an axisymmetric cavity. Journal of Sound and Vibration 87, 371-382.

STONEMAN, S. A. T., HOURIGAN, K., STOKES, A. N. \& WELSH, M. E. 1988 Resonant sound caused by flow past two plates in tandem in a duct. Journal of Fluid Mechanics 192, 455-484.

WILSON, T. A., BEAVERS, G. S., DECOSTER, M. A., HOLGER, D. K. \& REGENFUSS, M. D. 1971 Experiments on the fluid mechanics of whistling. Journal of the Acoustical Society of America 50, 366-372.

ZIADA, S. \& BÜHLMAN, E. T. 1992 Self-excited resonances of two-side-branches in close proximity. Journal of Fluids and Structures 6, 583-601.

ZIADA, S. \& SHINE, S. 1999 Strouhal numbers of flow-excited acoustic resonance of closed side branches. Journal of Fluids and Structures 13, 127-142. 


\section{FIGURE CAPTIONS}

Figure 1: Overview of pipe-cavity system and locations of pressure transducers.

Figure 2: Three-dimensional representations of pressure amplitude $p$ on a plane of frequency $f$ versus dimensionless pipe extension $\mathrm{X}_{\mathrm{T}} / \lambda$. Plots are shown for the three locations of the pressure transducers defined in Figure 1.

Figure 3: Plan views of three-dimensional plots of Figure 2. These views focus on the band of frequencies associated with generation of pronounced peaks of pressure amplitude $p$.

Figure 4: Variations of frequency $f_{P}$ and amplitude $P$ of predominant pressure peak as a function of length $X_{T}$ of pipe extension normalized by the pipe diameter $D$ and the acoustic wavelength $\lambda$. Also shown is the corresponding variation of the Quality (Q)-factor of the corresponding peaks in the pressure spectrum.

Figure 5: Selected spectra of the pressure $p$ as a function of length $X_{T}$ of the pipe extension normalized by the pipe diameter $\mathrm{D}$.

Figure 6: Zoomed-in plots of the predominant spectral peaks of Figure 5. Note that the amplitude scale of pressure $\mathrm{p}$ is adjusted to give approximately the same height in each plot, thereby providing an aid to interpreting the Quality (Q)-factor of the spectral peak.

Figure 7: Three-dimensional plots of the pressure amplitude $p$ on a plane of frequency $f$ and inflow velocity $U$ for two different values of pipe extension length $X_{\Upsilon}$ normalized by pipe diameter $\mathrm{D}$.

Figure 8: Variation of peak pressure amplitude $P$ and frequency of peak $f_{P}$ with inflow velocity $U$ for selected values of pipe extension length $X_{T}$ normalized by pipe diameter $D$.

Figure 9: Variations of Figure 8 plotted on the same set of axes.

Figure 10: Selected spectra of pressure $p$ for the data sets of Figures 8 and 9 . The value of velocity $U$ indicated on each plot corresponds to occurrence of the maximum value of peak amplitude $\mathrm{P}$ as $\mathrm{U}$ is varied.

Figure 11: Zoomed-in plots of spectral peaks corresponding to peak pressure amplitude $\mathrm{P}$ in the spectra of Figure 10. 

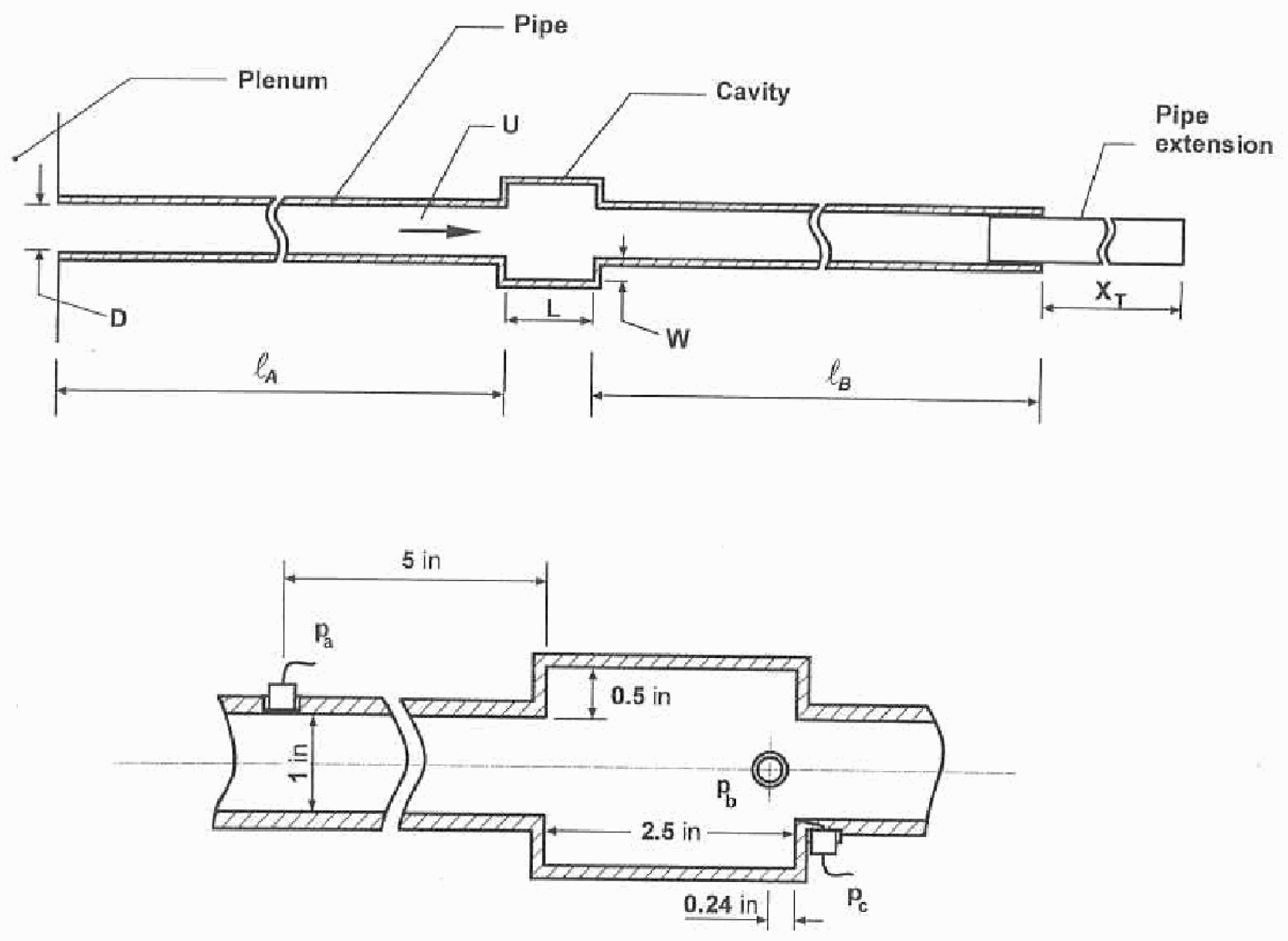

Figure 1: Overview of pipe-cavity system and locations of pressure transducers. 


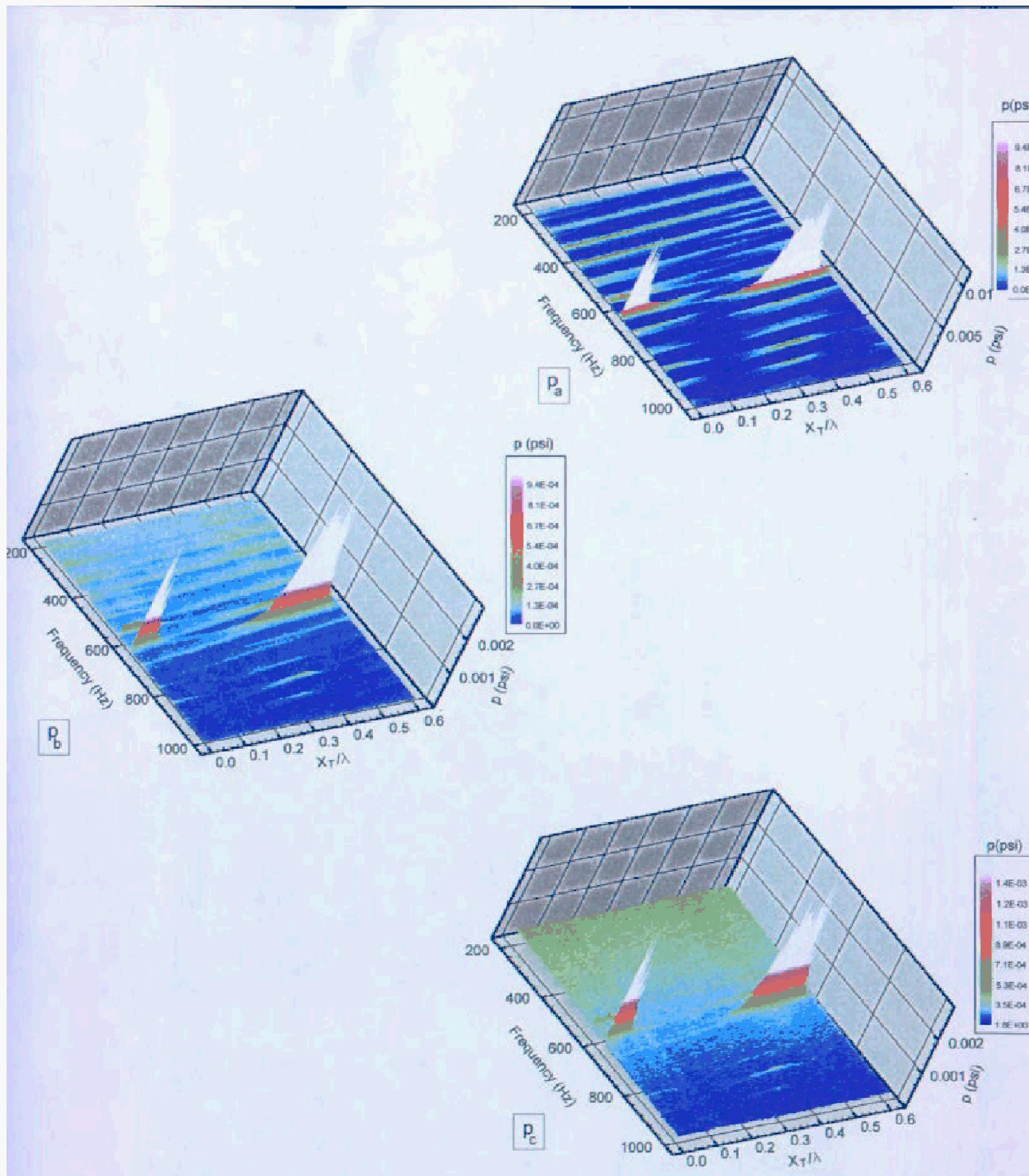

Figure 2: Three-dimensional representations of pressure amplitude $p$ on a plane of frequency $f$ versu dimensionless pipe extension $X_{r} / \lambda$. Plots are shown for the three locations of the pressure transducers defined Figure 1. 


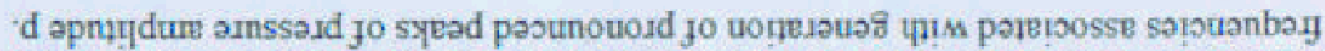

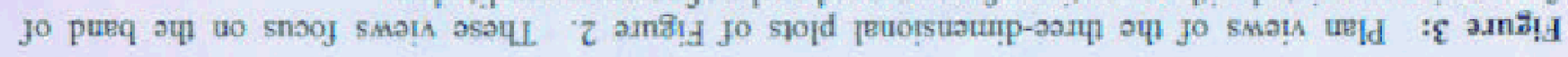
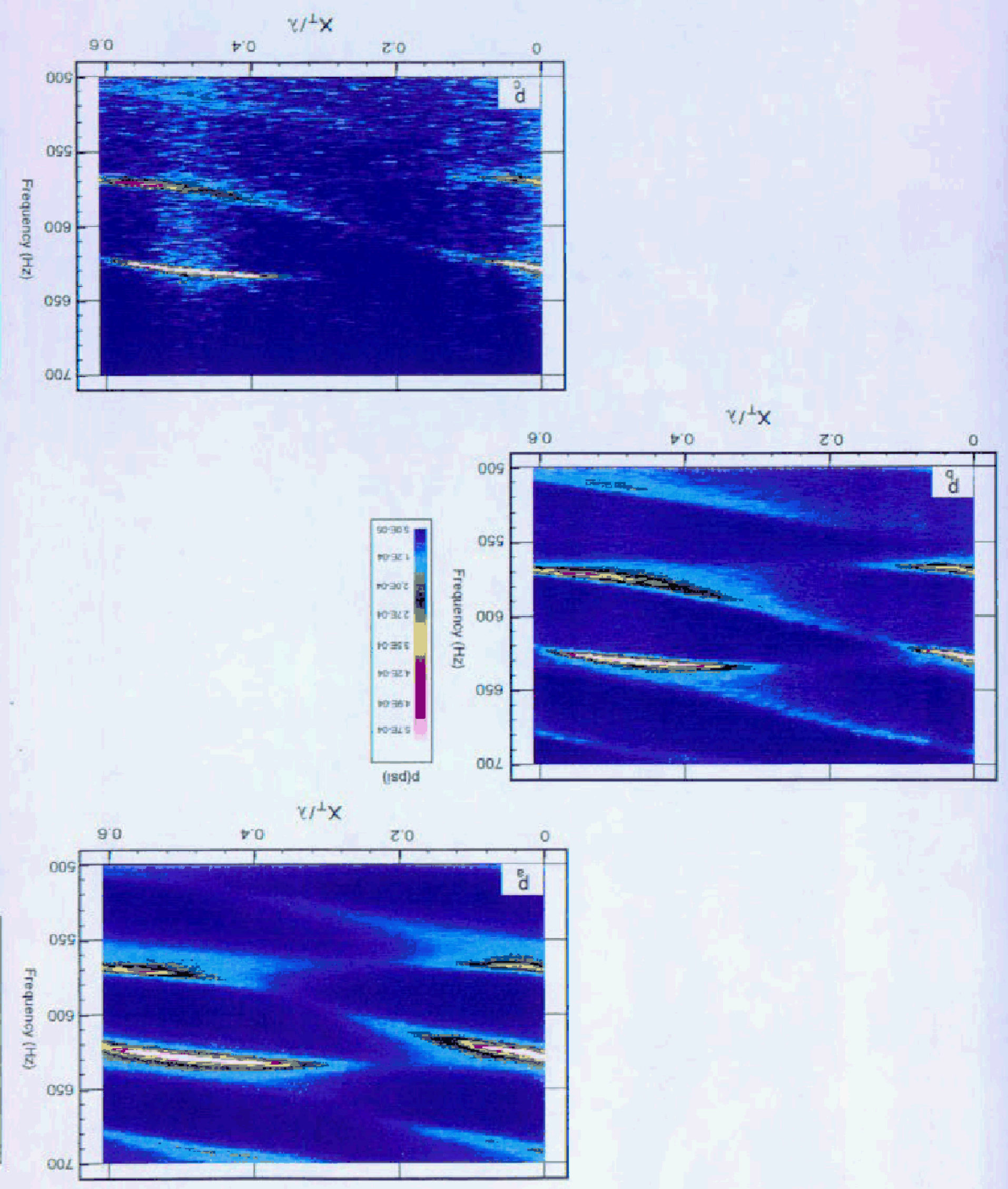

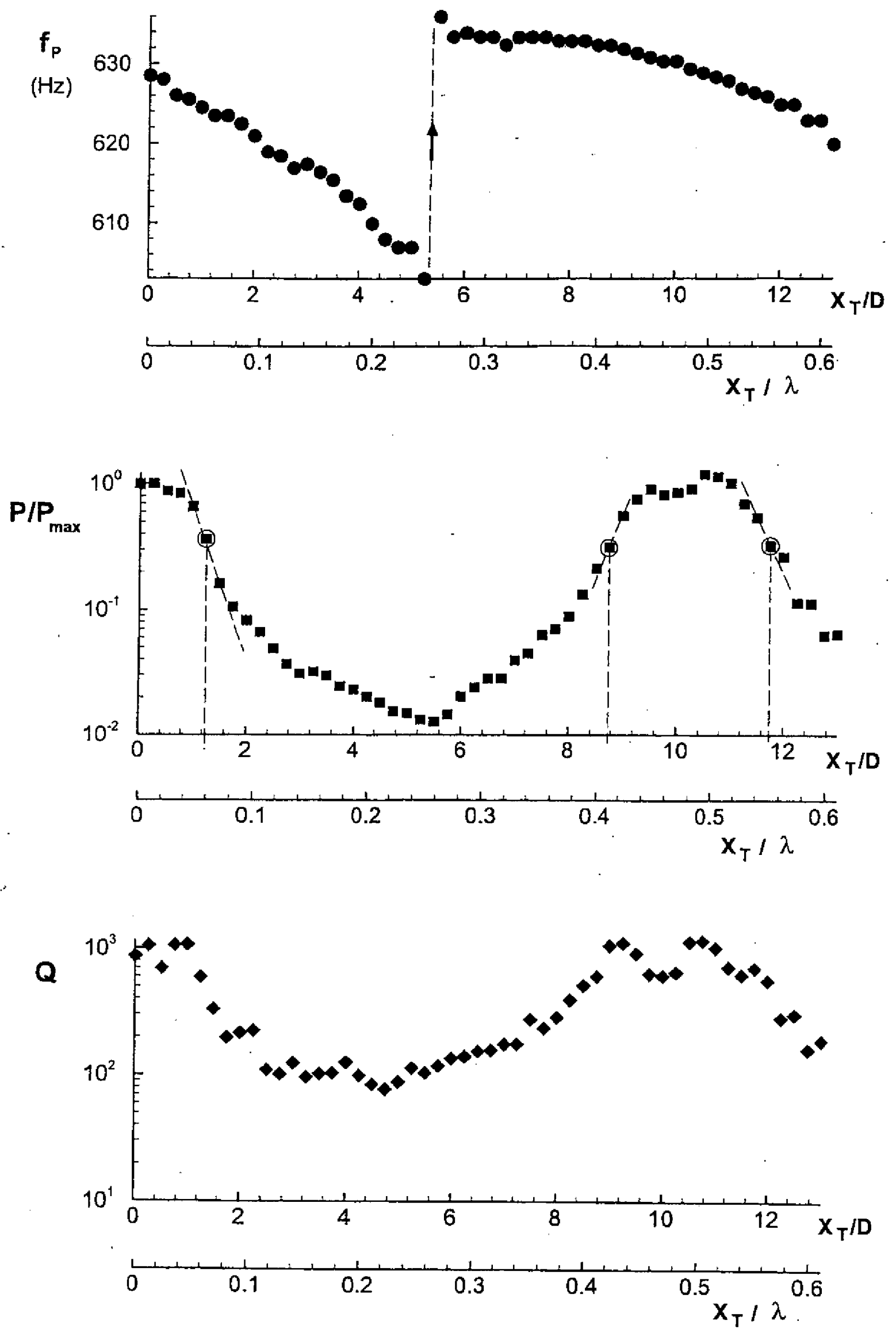

Figure 4: Variations of frequency $f_{P}$ and amplitude $P$ of predominant pressure peak as a function of length $X_{T}$ of pipe extension normalized by the pipe diameter $D$ and the acoustic wavelength $\lambda$. Also shown is the corresponding variation of the Quality (Q)-factor of the corresponding peaks in the pressure spectrum. 

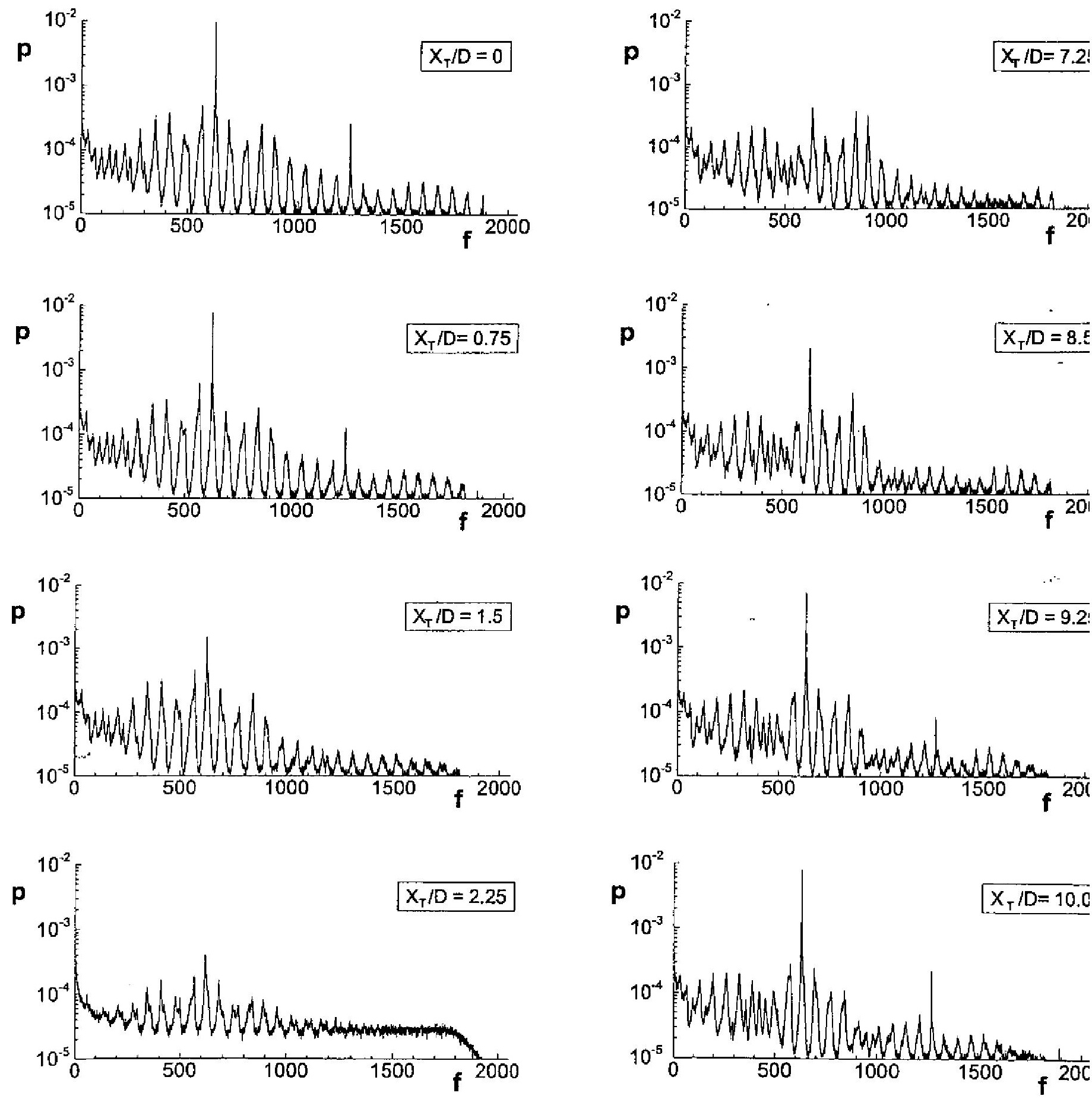

Figure 5: Selected spectra of the pressure $\mathrm{p}$ as a function of length $X_{\mathrm{T}}$ of the pipe extension normalized by the diameter D. 

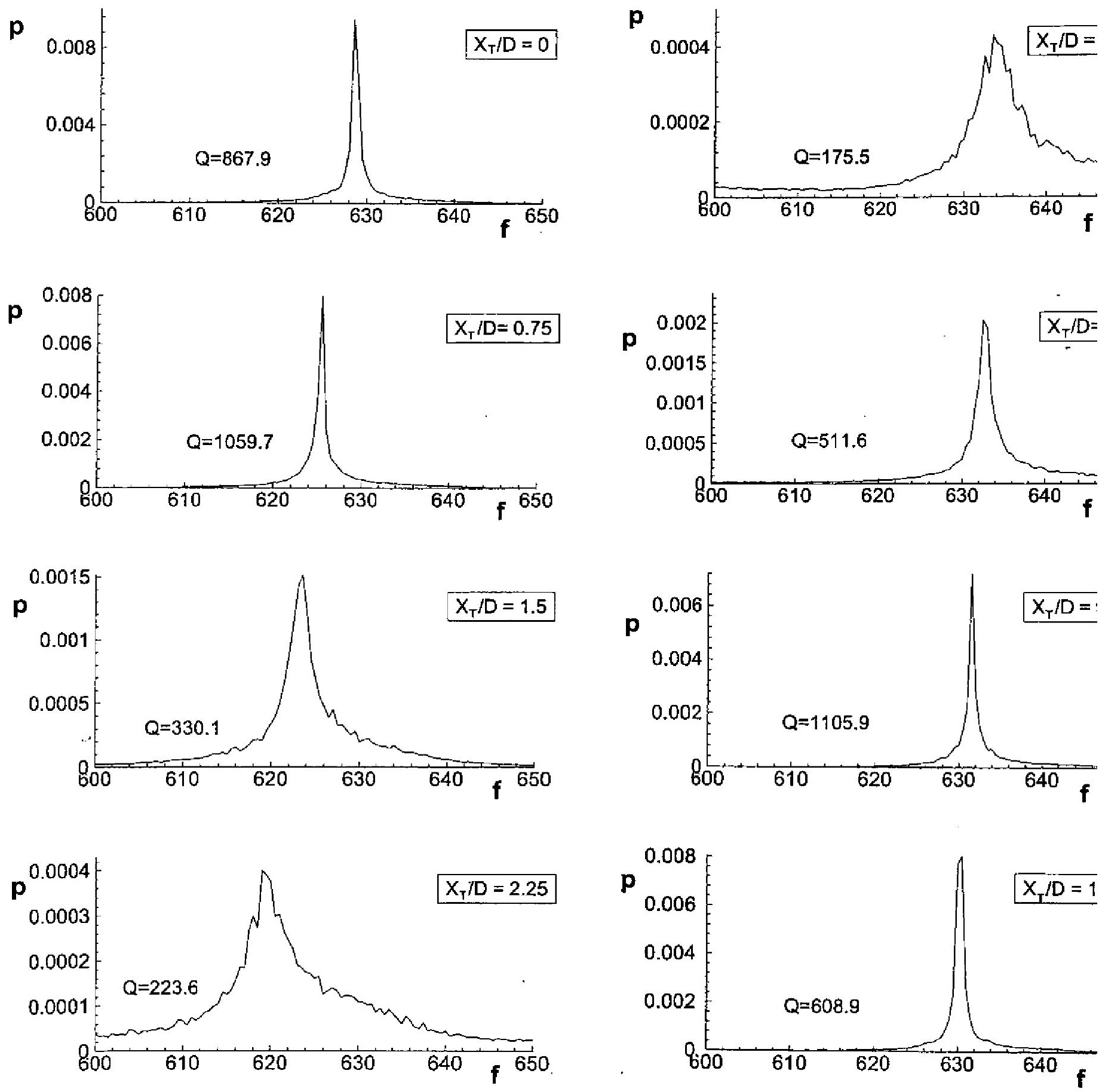

Figure 6: Zoomed-in plots of the predominant spectral peaks of Figure 5. Note that the amplitude scale of pressure is adjusted to give approximately the same height in each plot, thereby providing an aid to interpreting the Quality (C factor of the spectral peak. 

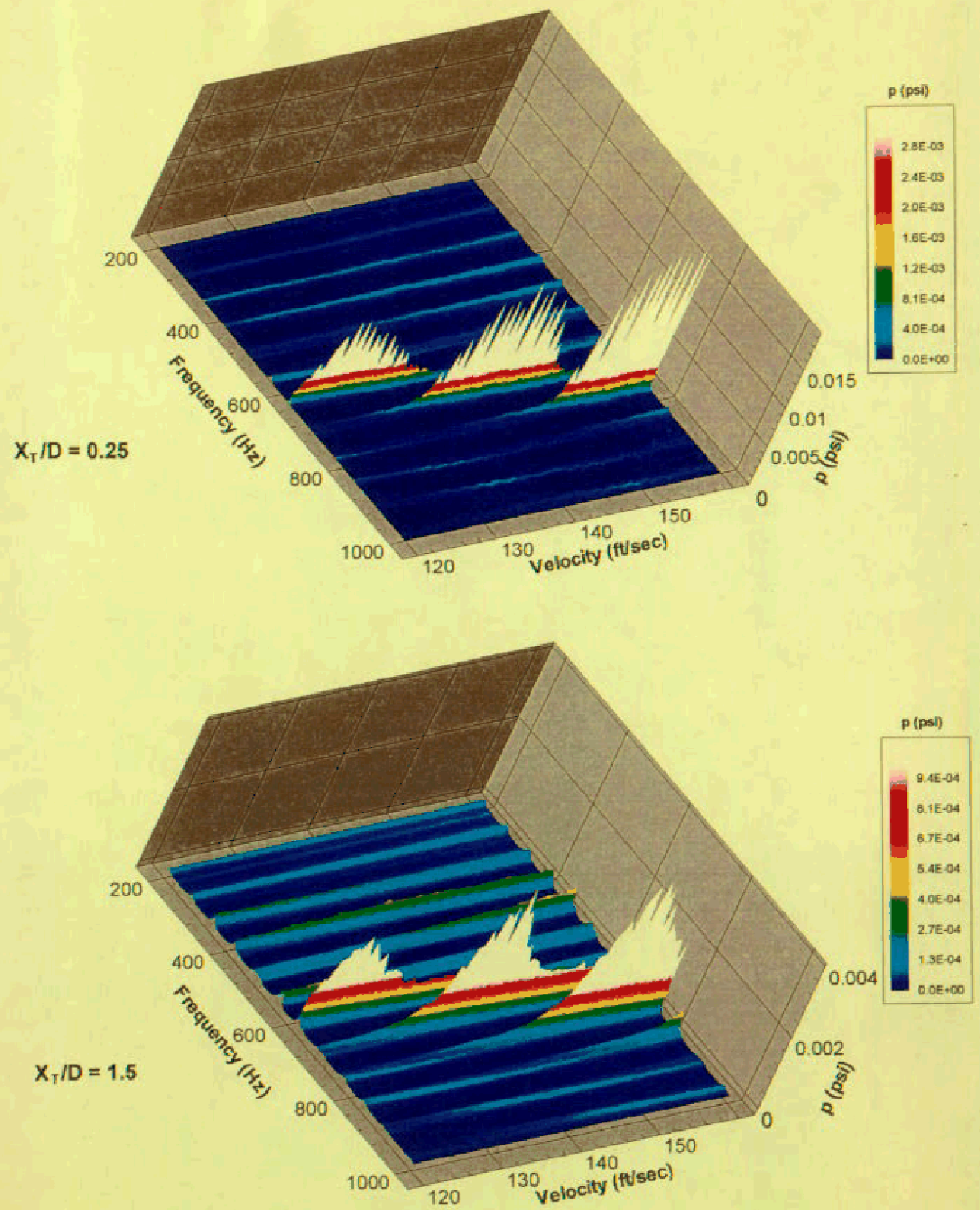

Figure 7: Three-dimensional plots of the pressure amplitude $p$ on a plane of frequency $f$ and inflow velocity $U$ for tv different values of pipe extension length $\mathrm{X}_{\mathrm{s}}$ normalized by pipe diameter $\mathrm{D}$. 

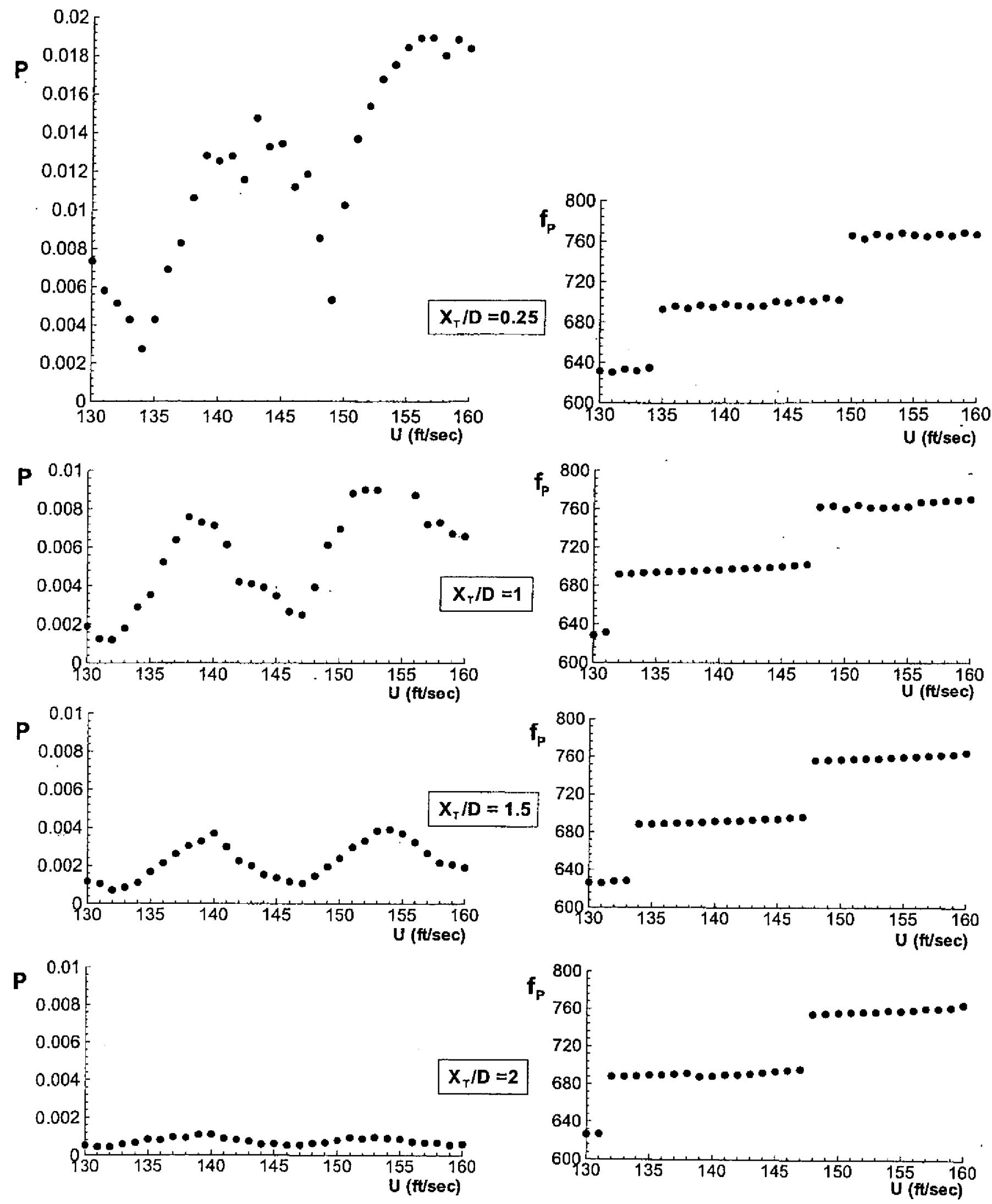

Figure 8: Variation of peak pressure amplitude $P$ and frequency $f_{p}$ of peak with inflow velocity $U$ for selected valut of pipe extension length $X_{T}$ normalized by pipe diameter $D$. 

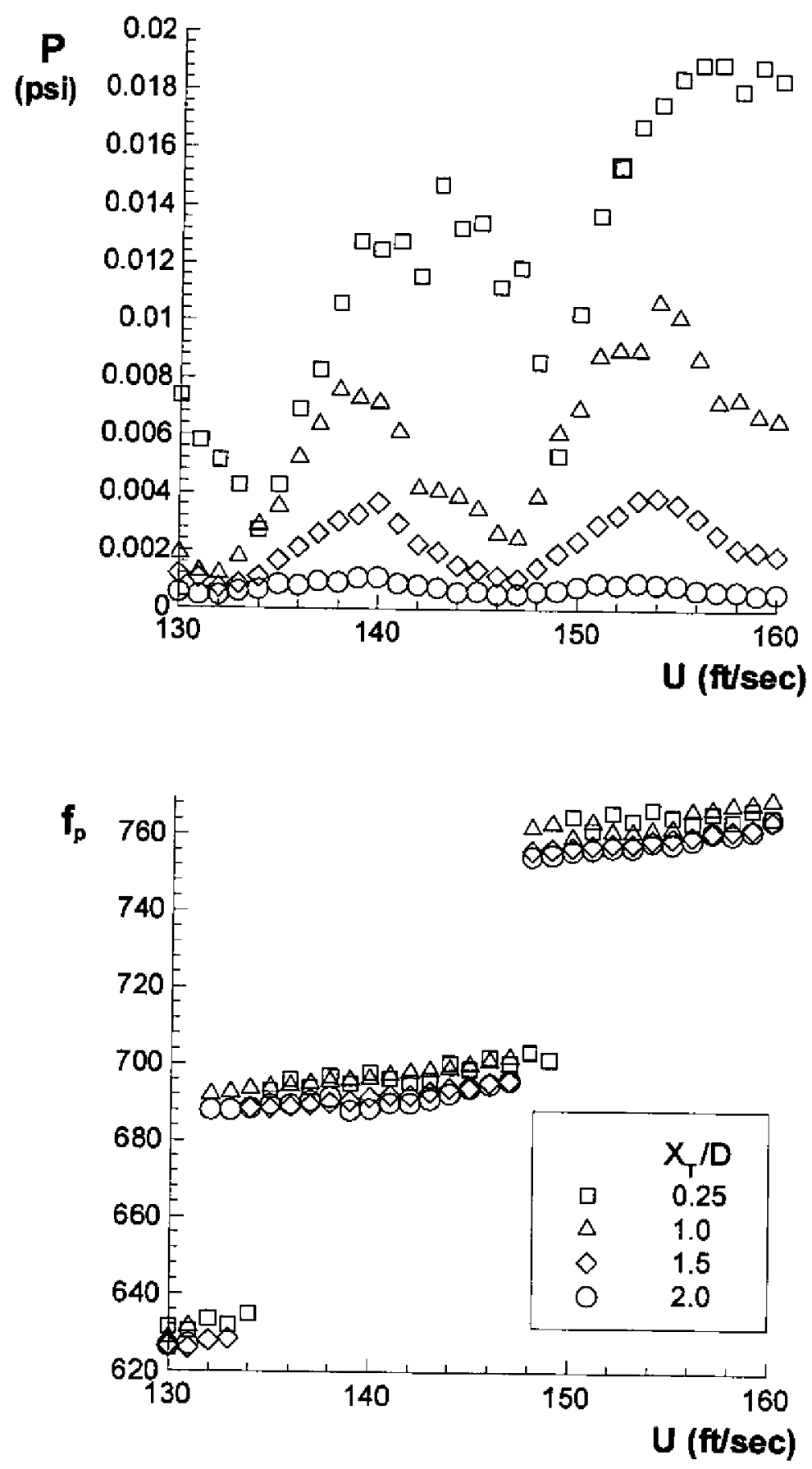

Figure 9: Variations of Figure 8 plotted on the same set of axes. 

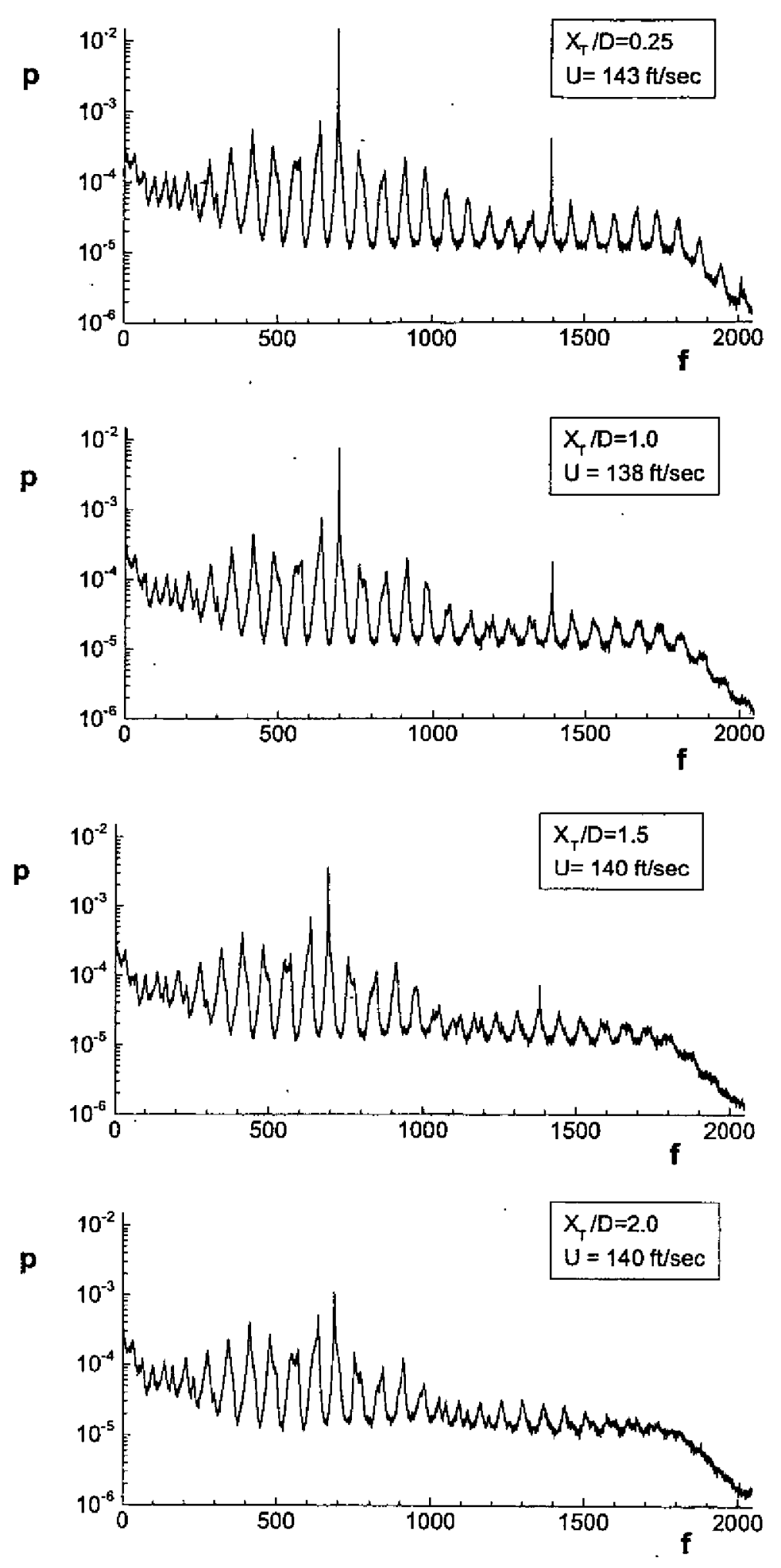

Figure 10: Selected spectra of pressure $p$ for the data sets of Figures 8 and 9 . The value of velocity $U$ indicated each plot corresponds to occurrence of the maximum value of peak amplitude $\mathrm{P}$ as $\mathrm{U}$ is varied. 

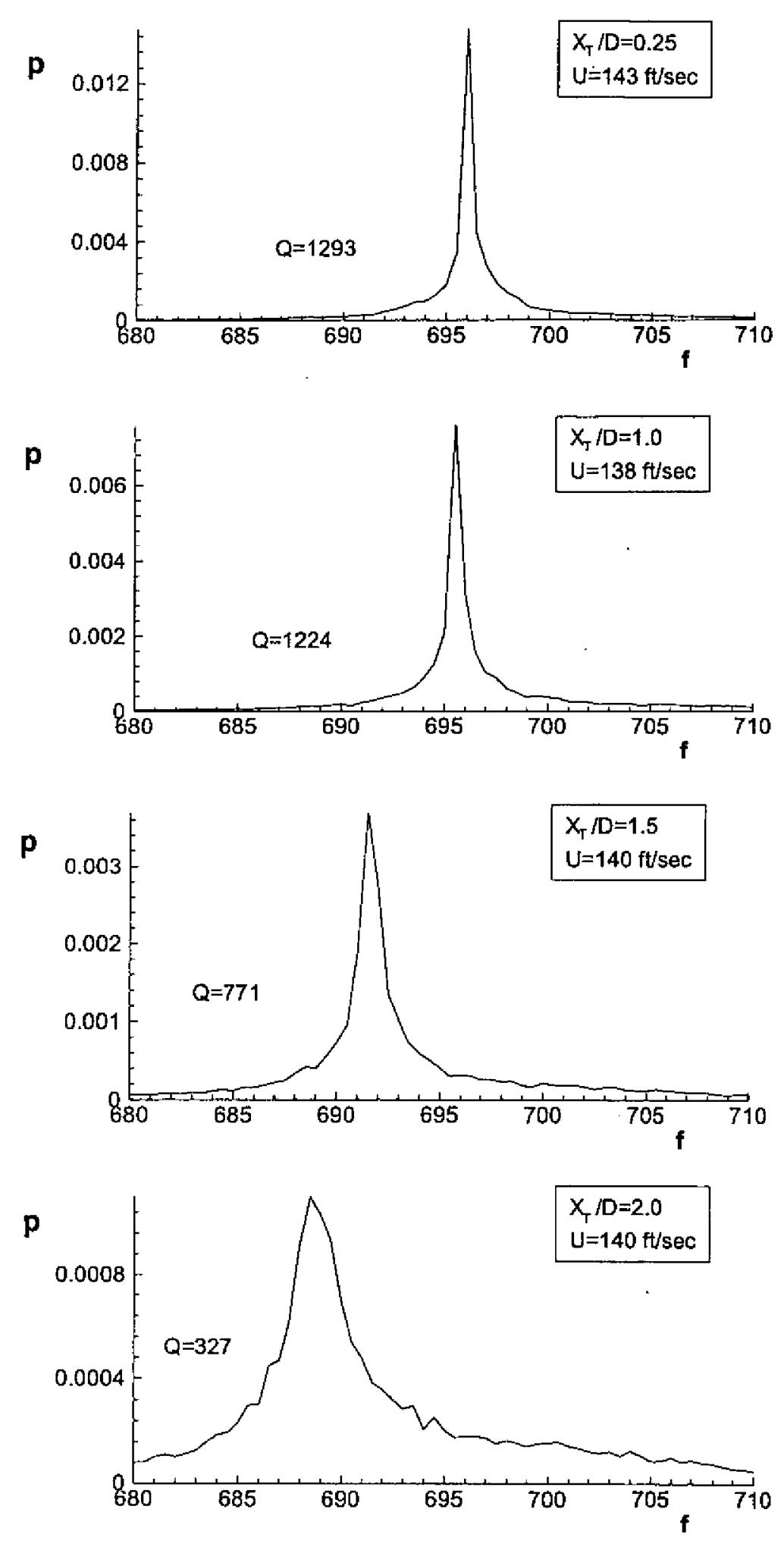

Figure 11: Zoomed-in plots of spectral peaks corresponding to peak pressure amplitude $P$ in the spectra of Figure 10 